\title{
Does body mass index influence clinical outcomes in patients with atrial fibrillation who receive direct oral anticoagulant therapy
}

\author{
(DIvana Jurin*, \\ (D) Marko Lucijanić, \\ (D)Anđela Jurišić, \\ (D)Aleksandar Blivajs, \\ (DB)Boris Starčević, \\ (DIrzal Hadžibegović
}

University Hospital Dubrava, Zagreb, Croatia
KEYWORDS: obese, atrial fibrillation, direct oral anticoagulant therapy. CITATION: Cardiol Croat. 2021;16(1-2):67. | https://doi.org/10.15836/ccar2021.67

*ADDRESS FOR CORRESPONDENCE: Ivana Jurin, Klinička bolnica Dubrava, Avenija Gojka Šuška 6, HR-10000 Zagreb, Croatia. / Phone: +385-98-559387 / E-mail: ivanajurin1912@gmail.com

ORCID: Ivana Jurin, https://orcid.org/0000-0002-2637-9691 • Marko Lucijanić, https://orcid.org/0000-0002-1372-2040 Anđela Jurišić, https://orcid.org/0000-0001-8316-4294 • Aleksandar Blivajs, https://orcid.org/0000-0003-3404-3837 Boris Starčević, https://orcid.org/0000-0002-3090-2772 • Irzal Hadžibegović, https://orcid.org/0000-0001-9139-5009

||||||||||||||||||||||||||||||||||||||||||||||||||||||||||||||||||||||||||||||||||||||||||||||||||||||||||||||||||||||||

Introduction: Randomized controlled trials of direct oral anticoagulant therapy (DOAC) included limited number of obese patients providing uncertainty about their efficacy in this population. ${ }^{1}$ Since DOACs are expected to be increasingly used, more information about efficacy and safety, especially among obese patients which might be exposed to suboptimal drug levels, is urgently needed. Therefore, we aimed to investigate in the real-life setting whether DOAC anticoagulated patients with atrial fibrillation stratified according to the different body mass index (BMI) subgroups experience different risks of unwanted outcomes.

Patients and Methods: We retrospectively investigated a real-life cohort of 325 DOAC anticoagulated patients with atrial fibrillation [179 receiving dabigatran (55\%), 74 apixaban (23\%) and 72 rivaroxaban (22\%)]. Patients were stratified according to the body mass index (BMI) into non-obese (233 with BMI $<30 \mathrm{~kg} / \mathrm{m}^{2}$ ), class I obesity (71 with BMI $30-34.9 \mathrm{~kg} / \mathrm{m}^{2}$ ) and class II + obesity ( 21 with BMI $\geq 35 \mathrm{~kg} / \mathrm{m}^{2}$ ).

Results: Patients with higher BMI receiving DOACs were more likely to experience stroke/systemic embolism sooner $(\mathrm{P}=0.043)$, experience major bleeding sooner $(\mathrm{P}<0.001)$ and have shorter time to composite event consisting of thrombosis, bleeding or death $(\mathrm{P}<0.001)$ whereas there was no significant association with overall survival $(\mathrm{P}=0.470)$. BMI was significantly associated with thrombosis but not bleeding among dabigatran treated patients, and significantly associated with bleeding but not thrombosis among patients treated with factor Xa inhibitors. Associations of higher thrombotic, bleeding and composite endpoint risks with higher BMI remained statistically significant in multivariate Cox regression models adjusted for age, gender, eGFR, CHA2DS2VASC and HAS-BLED.

Conclusion: Our findings indicate that obese patients receiving DOACs, especially ones with class II + obesity, might be under higher risks of stroke/bleeding depending on DOAC subtype. Loss of efficacy might be associated with dabigatran, whereas higher risk of major bleeding might be associated with factor Xa inhibitors.

LITERATURE IIIIIIIIIIIIIIIIIIIIIIIIIIIIIIIIIIIIIIIIIIIIIIIIIIIIIIIIIIIIIIIIIIIIIIIIIIIIIIIIIIIIIIIIIIIIIIIIIII

1. McCaughan GJB, Favaloro EJ, Pasalic L, Curnow J. Anticoagulation at the extremes of body weight: choices and dosing. Expert Rev Hematol. 2018 oct:11(10):817-828. https://doi.org/10.1080/17474086.2018.1517040 